\title{
A New Algorithm of Tracking Ballistic Missiles
}

\author{
Lifeng $\mathrm{Qi}^{1, \mathrm{a}}$, Guanglong Jiao ${ }^{1, \mathrm{~b}}$,ChuangMing Tong \\ ${ }^{1}$ School of Air and Missile Defense of Air Force Engineering University, Xi'an, 710051, China \\ aemail: qlf2009@sina.com, bemail: jgl2012@sina.com
}

Keywords: Ballistic issiles;Target tracking;Maximum likelihood estimator(ML);Probabilistic data association (PDA)

\begin{abstract}
A new algorithm for the problem of initiating the tracks assuming the target trajectories follow a deterministic state propagation, through the maximum likelihood (ML) estimator combined with the probabilistic data association (PDA) First some well-known approaches for statistical model selection and the advantage of using the MDL criterion were reviewed, Then a one-dimensional example was used to illustrate the MDL criterion for multiple composite hypothesis testing and the performance limit of the ML-PDA for track initiation interpreted in terms of the sharpness of the hypothesis testing. Finally, The MDL approach for the detection and initiation of tracks of incoming tactical ballistic missiles in the exo-atmospheric phase was applied using a surface based electronically scanned array (ESA) radar. The targets were characterized by low SNR, which lead to low detection probability and high false alarm rate. A batch of radar scans were processed to detect the presence of up to two targets. The ML-PDA estimator was used to initiate the tracks assuming the target trajectories follow a deterministic state propagation.
\end{abstract}

\section{Introduction}

The main focus of the ballistic missile acquisition is to initialize the missile trajectories using the measurements obtained by a surface-based radar for a short period of time (typically less than 10s) before the missile reentry phase. The radar beam pointing directions are assumed to cover a specific "cueing region" (taken here as $(30 \mathrm{~km}) 3$ ) provided by some early warning. The measurements obtained by the radar require a monopulse processing technique to obtain the full position measurements with associated variances. Due to low SNR and thus the low detection threshold to obtain a reasonable detection probability (around 0.6 for a dwell), the set of measurements contains a large number of false alarms (detection threshold exceedances due to noise).In addition, the number of targets within the cueing region is unknown. A track initiation algorithm has to determine how many targets are in the cueing region (model selection problem). If there are targets, the initial state of each target has to be estimated. In the following subsections, we present the target and radar model and the ML-PDA algorithm for missile trajectory parameter estimation. The detailed missile trajectory, radar model and the monopulse processing technique can be found in [1].

\section{Coordinate system and target trajector}

The motion of a missile above the atmosphere is governed by Kepler's laws. The earth centered inertial (ECI) coordinate system is used for the state propagation of the targets. This coordinate system has its origin at the center of the earth, with the positive $\mathrm{x}$ axis pointing along the vernal equinox and the positive $\mathrm{z}$ axis to the north pole ${ }^{[2]}$. The radar and target locations are better interpreted in the earth centered earthfixed (ECEF) coordinate system. This coordinate system has its origin at the center of the earth, with the positive $\mathrm{x}$ axis passing through the prime meridian at the equator and the positive $\mathrm{z}$ axis passing through the north pole. Let $X_{k}=\left[\begin{array}{lll}r_{k} & \dot{r}_{k}\end{array}\right]^{\prime}$ be the 6-dimensional target state vector at time $t_{k}$, where the vectors $r_{k}=\left[\begin{array}{llll}\xi\left(t_{k}\right) & \eta\left(t_{k}\right) & \varsigma & \left(t_{k}\right)\end{array}\right]^{\prime}$ and 
$\dot{r}_{k}=\left[\begin{array}{lll}\dot{\xi}\left(t_{k}\right) & \dot{\eta}\left(t_{k}\right) & \dot{\zeta}\left(t_{k}\right)\end{array}\right]^{\prime}$ are the position and velocity of the target in ECI coordinates, respectively. Given the initial state $X_{0}$ of the target at time $t_{0}$, the state at $t_{k}$ is $X_{k}=\left[r_{k}\left(X_{0}, t_{0}, t_{k}\right)^{\prime} \quad \dot{r}_{k}\left(X_{0}, t_{0}, t_{k}\right)^{\prime}\right]^{\prime}$ where $r_{i}()$ and $\dot{r}_{i}()$ are determinstic functions given in[1].

Another coordinate system is located at the radar. This coordinate has its origin at the radar, with positive $\mathrm{x}$ pointing toward north and positive $\mathrm{z}$ axis pointing $\mathrm{up}^{[3]}$. It is often called the local Cartesian frame of radar or ENU frame indicating the axes pointing toward East, North and Up. Given the radar position Oradar in ECEF coordinates, the state of the target in radar coordinates is given by

$$
X_{k}^{\text {radar }}=A X I S\left(X_{k}, \mathrm{O}^{\text {radar }}\right)
$$

where $\operatorname{AXIS}(\bullet)$ is the coordinate conversion given in [1]. Figure 1 illustrates the missile trajectory and the cueing region. The state estimate of the target is obtained in radar coordinates and then converted into ECEF coordinates.

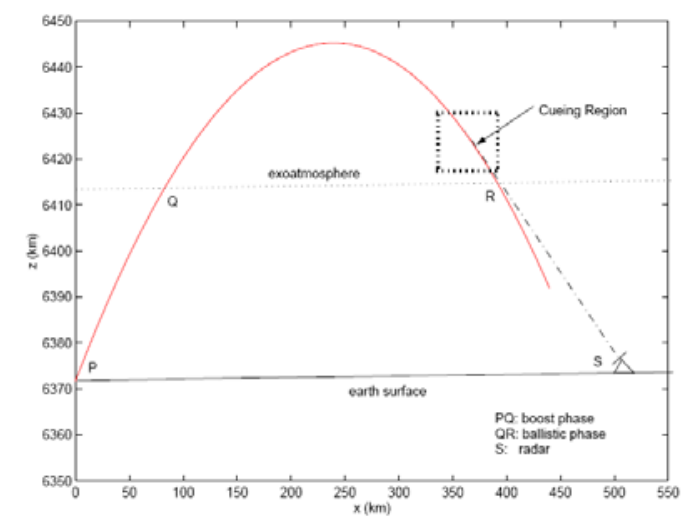

Figure 1: Illustration of the stages of a ballistic missile trajectory

\section{The Log-likelihood radio with Probabilistic data association}

We now present the maximum likelihood (ML) estimator combined with the probabilistic data association (PDA) technique to tackle the uncertainty of measurement origin. We assume that the radar receiver waits for $\mathrm{N}$ scans before a decision about track existence is made ${ }^{[4]}$. Thus we have $\mathrm{N}$ sets of measurements made at times $t_{i}, \mathrm{i}=1,2, \ldots, \mathrm{N}$ where the index $\mathrm{i}$ denotes the scan number. The $\mathrm{k}$-th measurement in the $\mathrm{i}$-th scan is defined by the vector

$$
Z_{i k}=\left[\begin{array}{llll}
\Re_{i k} & r_{i k} & \beta_{i k} & \mathcal{E}_{i k}
\end{array}\right]
$$

where $\mathfrak{R}_{i k}, r_{i k}, \beta_{i k}, \varepsilon_{i k}$ are the signal plus noise to noise ratio (SNNR), slant range, bearing and elevation, respectively. The SNNR is a target feature and it has been shown to reduce the data association uncertainty ${ }^{[8]}$. Its model is presented in sequel. The radar performs a volumetric search over the cueing region based on prior information. It is assumed that all the dwells in a scan are made at (practically) the same time. Denote by mi the number of measurements in the i-th scan obtained at time ti. The set is written as

$$
Z(i)=\left\{Z_{i k}\right\}_{k=1}^{m_{i}}
$$

The cumulative set of measurements during the entire period is

$$
Z=\{Z(i)\}_{i=1}^{N}
$$

Acceptance of the Estimate

We first consider the hypothesis test of one target vs. no target:

- $\mathrm{H}_{1}$ : There is one track corresponding to an existing target and $\hat{x}$ is the global maximum of the likelihood ratio

- $\mathrm{H}_{0}: \hat{x}$ does not correspond to a valid track

We use the log-likelihood ratio $\ln (\Lambda[Z, X])$ with the penalty terms given by the approximate 
MDL criterion as the test statistic for the hypothesis test of $\mathrm{H} 1$ against $\mathrm{H} 0$. Denote $\mathrm{T}(\mathrm{Z})$ as the test statistic. We have

$$
T(Z)=\ln (\Lambda[Z, \hat{x}])-\frac{k}{2} \ln \left(\frac{S_{N}}{2 \pi}\right)-\ln \int|I(X)|^{\frac{1}{2}} d x
$$

where $\mathrm{S}_{\mathrm{N}}$ is the number of measurements given by

$$
S_{N}=\sum_{i=1}^{N} m_{i}
$$

and $\mathrm{k}$ is the the dimension of the MLE $\hat{x}$ (in this case $\mathrm{k}=6$ ) which is

$$
\hat{x}=\arg \max \ln (\Lambda[Z, x])
$$

The parameter space $\Omega$ covers the cueing region and all possible velocity vectors of the missile $^{[5]}$. Without considering the information reduction factor, the integral $\int_{\Omega}|I(X)|^{\frac{1}{2}} d x$ is obtained numerically and can be computed off-line. We choose $\mathrm{H} 1$ when $T(Z)>0$.Alternatively, we can use $\log \Lambda[Z, \hat{x}]$ as the test statistic and compare it with a prespecified threshold for certain power based on the Gaussian approximation under H1. The numerical approach for obtaining the mean and variance of the test statistic under $\mathrm{H} 1$ can be found in [6]. We will compare the MDL with the GLRT in simulation.

If a track is accepted, the MLE $\hat{x}$ is used as the state estimate with the corresponding covariance given by approximate Cramer-Rao lower bound (CRLB) which will be presented in the next subsection. The estimator uses all the measurements $\mathrm{Z}$ to extract one track at a time. The measurements having the association probability greater than 0.5 to the presumed track at each scan are deleted after the track has been initiated. If a measurement at a specific scan is deleted, all the measurements from its adjacent beams (in the same range bin) are also deleted (if there is any) to compensate for the effect of multiple detections. It is very important to remove all measurements from the main beam and the adjacent beams after a track is declared. The number of false tracks decreases when using this procedure especially when the SNR is low. The rest of the measurements are used to extract another target using the same ML-PDA estimator with MDL model selection criterion $^{[7]}$. The procedure continues until the test statistic $\mathrm{T}(\mathrm{Z})$ is less than 0 .

Cramer-Rao Lower Bound of the Estimator

As discussed in [8], the Cramer-Rao lower bound (CRLB) is a lower limit on the variance that can be achieved by an unbiased estimator. The MLE can be said to be efficient if its sample variance is statistically commensurate with the CRLB. For the MLE $\hat{x}$ of the state $\mathrm{x}$, we have

$$
\varepsilon\left[(x-\hat{x})(x-\hat{x})^{\prime}\right] \geq J^{-1}
$$

To simplify the calculation, we also assume that the information reduction factor is a constant across different scans. Only those scans with at least one measurement in the gate are considered in obtaining the FIM. Thus we can write the FIM as

$$
\left.J=q\left(P_{D}, \lambda V_{g}, g, o \Sigma^{4}\right) \sum_{i=1}^{N}\left(\frac{1}{\left(\sigma^{r}\right)^{2}}\left[\nabla_{x} r_{i}(x)\right] \nabla_{x} r_{i}(x)\right]^{\prime}+\frac{1}{\left(\sigma^{\beta}\right)^{2}}\left[\nabla_{x} \beta_{i}(x)\right]\left[\nabla_{x} \beta_{i}(x)\right]^{\prime}+\frac{1}{\left(\sigma^{\varepsilon}\right)^{2}}\left[\nabla_{x} \varepsilon_{i}(x)\right]\left[\nabla_{x} \varepsilon_{i}(x)\right]^{\prime}\right)
$$

The standard deviations of the bearing and elevation measurements depend on the target position within the radar beam ${ }^{[8]}$. Assuming the target is uniformly distributed within the rectangular region covered by one radar beam, the average measurement noise for a target is $\sigma^{\beta}=\sigma^{\varepsilon}=0.0067 \mathrm{rad}$ when the detection threshold is 3.25. If the detection probability per scan is $P_{D}=P_{\text {Dave }}=0.464$, we have the average target return $\alpha \Sigma^{4}=5.76$. The false alarm probability is obtained by averaging the false detections in eight adjacent beams when the target is uniformly distributed within the rectangle area of the main beam (the one pointing to the target). Using the integral approach developed in [6], the information reduction factor is $q(\cdot)=0.346$ for a single target with Poisson distributed false alarms. This information reduction factor $q(\cdot)$ is averaged over 100 randomly generated target positions uniformly distributed within the rectangular region of the main beam. It approximately 
quantifies the estimation accuracy. For multiple target scenarios, we assume the approximate CRLB for a single target is still valid for quantifying the efficiency of the estimator. For Swerling III targets with $\mathrm{SNR}=6 \mathrm{~dB}$ at boresight and various detection thresholds, we list the detection probability, false alarm probability and the information reduction factor averaged over the above rectangular region in Table 1.

\begin{tabular}{|c|c|c|c|c|}
\hline Threshold T & $P_{F A}$ & $P_{D}$ at boresight & $P_{\text {Dave }}$ & Information reduction $q$ \\
\hline 3.15 & 0.112 & 0.586 & 0.483 & 0.3792 \\
\hline 3.10 & 0.115 & 0.593 & 0.489 & 0.3813 \\
\hline 3.05 & 0.119 & 0.599 & 0.496 & 0.3826 \\
\hline 3.00 & 0.122 & 0.605 & 0.503 & 0.3848 \\
\hline 2.95 & 0.126 & 0.611 & 0.509 & 0.3861 \\
\hline 2.90 & 0.130 & 0.617 & 0.516 & 0.3876 \\
\hline 2.85 & 0.134 & 0.624 & 0.523 & 0.3891 \\
\hline 2.80 & 0.138 & 0.630 & 0.530 & 0.3906 \\
\hline 2.75 & 0.143 & 0.636 & 0.537 & 0.3913 \\
\hline 2.70 & 0.147 & 0.643 & 0.544 & 0.3031 \\
\hline 2.65 & 0.151 & 0.656 & 0.551 & 0.3949 \\
\hline
\end{tabular}

Table 1: Detection thresholds used for the target with SNR=6dB at boresight and the corresponding information reduction factors.

\section{Simulation results}

In this section we consider a two-target scenario to illustrate the operation of the ML-PDA estimator under various SNRs. The scenario contains two Swerling III targets with the same

average RCS. We assume that the two targets enter the radar acquisition region at $\mathrm{t}=0 \mathrm{~s}$ with the initial state $r_{0}^{1 \text { ture }}=\left[\begin{array}{llll}720.0 & 6050.0 & 2180.0\end{array}\right]^{\prime} \mathrm{km}, \dot{r}_{0}^{1 \text { ture }}=\left[\begin{array}{llll}-0.045 & -1.531 & -0.694\end{array}\right]^{\prime} \mathrm{km} / \mathrm{s}$, and $r_{0}^{2 \text { ture }}=\left[\begin{array}{lll}718.0 & 6052.0 & 2179.5\end{array}\right]^{\prime} \mathrm{km}, \quad \dot{r}_{0}^{2 \text { ture }}=\left[\begin{array}{lll}-0.045 & -1.541 & -0.695\end{array}\right]^{\prime} \mathrm{km} / \mathrm{s}$; the radar position

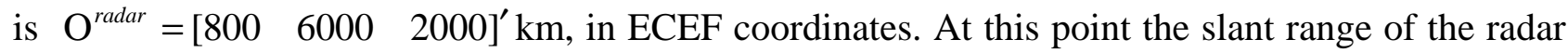
to target 1 is $204.1 \mathrm{~km}$ and the slant range of radar to target 2 is $203.2 \mathrm{~km}$.

It is assumed that the radar cueing region for initial radar beam pointing is available from prior information. The radar cueing volume is approximately $(30 \mathrm{~km}) 3$ and the radar beam packing is 7 by 7 rectangular with each dwell consisting 330 bins of length $90 \mathrm{~m}$. The average output SNR at boresight for each target is $6 \mathrm{~dB}$ to $8 \mathrm{~dB}$ for the cases considered. Notice that the average SNR in a resolution cell is $4.4 \mathrm{~dB}$ when the target SNR at boresight is $6 \mathrm{~dB}$. The detection threshold for acquisition of $6 \mathrm{~dB}$ targets is $\tau=2.87$, yielding an average detection probability 0.52 . The spatial density of false alarms in the validation gate is dorminated by the target-due extraneous detections in the eight adjacent beams when the target is uniformly distributed within the rectangular region centered at the main beam.5 With the parameter settings specified as above we have $\lambda V_{g}=1.2$. The radar scan rate is $10 \mathrm{~Hz}$. The total number of scans $\mathrm{N}$ varies from 30 to 60 . The scenarios are denoted as H0: no target is present; H1: only target 1 is present; H2: both target 1 and target 2 are present. We are interested in the probability of choosing the correct model under each case. The target SNR at boresight varies from $6 \mathrm{~dB}$ to $8 \mathrm{~dB}$. In each case, the detection threshold is chosen to maintain $\lambda V_{g} \leq 1.2$.

The results from 100 Monte Carlo runs for three scenarios (H0, H1, H2) are listed in Table 1. When two tracks are initiated, the track to truth association is based on the geometric distance between the true and estimated state. If the distance is greater than three times the measurement standard deviation, the track is declared as false. If we want the probability of choosing the correct model to be greater than 0.95 for $\mathrm{N}=60$, the target SNR has to be greater than $6 \mathrm{~dB}$ at boresight (or 
equivalently,the average SNR has to be greater than $4.4 \mathrm{~dB}$ ). When the target SNR increases, the performance of the MDL model selection also improves.

For the same scenarios, we also use the GLRT with a threshold to obtain 95\% acquisition probability under $\mathrm{H} 1$ for a single target (based on the Gaussian approximation as in[8]). The results are listed in Table 2. We can see that the GLRT yields lower acquisition probabilities under both one-target and two-target scenarios compared with the MDL approach. This is because the threshold chosen by obtaining 95\% target acquisition probability is not optimal in comparison with the penalty terms used in MDL.

The CRLB and the sample standard deviation for the two-target scenario are listed in Table 2 with $\mathrm{N}=50$ and $\tau=2.87$ for the $6 \mathrm{~dB}$ target and $\tau=3.63$ for the $8 \mathrm{~dB}$ target. In most cases, the sample standard deviation is slightly larger than the approximate CRLB. This can be explained by several factors: a) the false alarms are not uniformly distributed in the cueing region; b) the range measurement noise is not Gaussian; c) the target trajectories used in simulation yield different off-boresight angles from scan to scan and could be better than the average CRLB. The results are acceptable in view of the fact that they are based on a 100 run average, which allow $\pm 14 \%$ error within the $95 \%$ confidence region.

\begin{tabular}{|r|r|c|c|c|c|c|}
\hline SUN & $\begin{array}{c}\text { Parameter } \\
\text { of target 1 }\end{array}$ & $\hat{\sigma}$ & $\sigma$ CRLB & $\begin{array}{c}\text { Parameter } \\
\text { of target 2 }\end{array}$ & $\hat{\sigma}$ & $\sigma$ CRLB \\
\hline $6 \mathrm{~dB}$ & $\xi(\mathrm{m})$ & 698.8 & 755.6 & $\xi(\mathrm{m})$ & 720.2 & 754.4 \\
\hline $6 \mathrm{~dB}$ & $\eta(\mathrm{m})$ & 1012.2 & 860.9 & $\eta(\mathrm{m})$ & 965.3 & 862.2 \\
\hline $6 \mathrm{~dB}$ & $\varsigma(\mathrm{m})$ & 398.2 & 371.7 & $\varsigma(\mathrm{m})$ & 408.4 & 381.6 \\
\hline $6 \mathrm{~dB}$ & $\dot{\xi}(\mathrm{m} / \mathrm{s})$ & 214.3 & 233.6 & $\dot{\xi}(\mathrm{m} / \mathrm{s})$ & 219.6 & 233.3 \\
\hline $6 \mathrm{~dB}$ & $\dot{\eta}(\mathrm{m} / \mathrm{s})$ & 297.8 & 265.5 & $\dot{\eta}(\mathrm{m} / \mathrm{s})$ & 317.1 & 265.9 \\
\hline $6 \mathrm{~dB}$ & $\dot{\zeta}(\mathrm{m} / \mathrm{s})$ & 138.4 & 114.3 & $\dot{\zeta}(\mathrm{m} / \mathrm{s})$ & 130.6 & 117.4 \\
\hline $8 \mathrm{~dB}$ & $\xi(\mathrm{m})$ & 628.3 & 648.9 & $\xi(\mathrm{m})$ & 650.6 & 647.9 \\
\hline $8 \mathrm{~dB}$ & $\eta(\mathrm{m})$ & 864.6 & 739.3 & $\eta(\mathrm{m})$ & 901.5 & 740.4 \\
\hline $8 \mathrm{~dB}$ & $\varsigma(\mathrm{m})$ & 310.4 & 319.2 & $\varsigma(\mathrm{m})$ & 346.3 & 327.7 \\
\hline $8 \mathrm{~dB}$ & $\dot{\xi}(\mathrm{m} / \mathrm{s})$ & 209.7 & 200.6 & $\dot{\xi}(\mathrm{m} / \mathrm{s})$ & 210.5 & 200.3 \\
\hline $8 \mathrm{~dB}$ & $\dot{\eta}(\mathrm{m} / \mathrm{s})$ & 267.8 & 228.0 & $\dot{\eta}(\mathrm{m} / \mathrm{s})$ & 268.1 & 228.3 \\
\hline $8 \mathrm{~dB}$ & $\dot{\zeta}(\mathrm{m} / \mathrm{s})$ & 88.7 & 98.1 & $\dot{\zeta}(\mathrm{m} / \mathrm{s})$ & 96.9 & 100.8 \\
\hline
\end{tabular}

Table 2: CRLB and the parameter estimates' sample standard deviations for the two-target scenario with various SNRs

\section{Conclusion}

In this thesis the track initiation problem was formulated as multiple composite hypothesis testing using maximum likelihood estimation with probabilistic data association (ML-PDA). The number of tracks was determined based on the minimum description length (MDL) criterion. We 
first reviewed some well-known approaches for statistical model selection and the advantage of using the MDL criterion. Then a one-dimensional example was used to illustrate the MDL criterion for multiple composite hypothesis testing and the performance limit of the ML-PDA for track initiation interpreted in terms of the sharpness of the hypothesis testing. Finally, We applied the MDL approach for the detection and initiation of tracks of incoming tactical ballistic missiles in the exo-atmospheric phase using a surface based electronically scanned array (ESA) radar. The targets were characterized by low SNR, which lead to low detection probability and high false alarm rate. A batch of radar scans were processed to detect the presence of up to two targets. The ML-PDA estimator was used to initiate the tracks assuming the target trajectories follow a deterministic state propagation. The approximate MDL criterion was used to determine the number of valid tracks in a surveillance region. The detector/estimator was shown to be efficient even at 4.4dB average SNR (within the beam, i.e., in a resolution cell).

\section{References}

[1] H. Chen, T. Kirubarajan, and Y. Bar-Shalom, "Multiple Low Observable Ballistic Missile Track Initiation Using an ESA Radar” , ESP Lab Tech Report, University of Connecticut, March 2001.

[2] F. Daum, “A System Approach to Multiple Target Tracking”, in Multitarget-Multisensor Tracking: Applications and Advances II, Y. Bar-Shalom, Ed., 1992.

[3] O. E. Drummond, “Track Fusion with Feedback” , Proc. of SPIE Conf. on Signal and Data Processing of Small Targets, Vol. 2759, pp. 342-360, April 1996.

[4] R. Helmick, “IMM Estimator with Nearest-Neighbor Joint Probabilistic Data Association” , in Multitarget-Multisensor Tracking: Applications and Advances,Y. Bar-Shalom and W. D. Blair, Eds., 2000.

[5] C. Jauffret, and Y. Bar-Shalom, "Track Formation with Bearing and Frequency Measurements in Clutter" IEEE Transactions on Aerospace and Electronic Systems, pp. 999-1010, November 1990.

[6] S. Kay, Fundamentals of Statistical Signal Processing, Vol. II-Detection Theory, Prentice Hall, 1998.

[7] T. Kirubarajan, and Y. Bar-Shalom, "Low Observable Target Motion Analysis Using Amplitude Information”, IEEE Transactions on Aerospace and Electronic Systems, 32(3), pp. 1367-1384, July 1996.

[8] X. Li, Z-Q. Luo, K. M. Wong and E. Bosse, “An Interior Point Linear Programming Approach to Two-Scan Data Association” , IEEE Trans. Aerospace and Electronic Systems, 35(2), pp. 474-490, 1999. 